\title{
STUDIES ON THERMOREVERSIVE MUCOADHESIVE OPHTHALMIC IN SITU GEL OF AZITHROMYCIN
}

\author{
Bhatia Honey Bala $^{1}$, Sachan Ajay ${ }^{2}$, Bhandari Anil ${ }^{3}$ \\ ${ }^{1}$ Algen Exports, 487/65, National Market, Peeragarhi, New Delhi, India \\ ${ }^{2}$ Central Drugs Standard Control Organization, New Delhi, India \\ ${ }^{3}$ Faculty of Pharmaceutical Sciences, Jodhpur National University, Jodhpur-342001, Rajasthan, India \\ *Corresponding Author, E-mail: algen.healthcare@gmail.com, hbhatia@algen.co.in
}

\begin{abstract}
In the present work, stability studies, microbiological study, ocular irritation studies and ocular permeation studies were performed on prepared thermoreversible gels of Azithromycin. Franz-diffusion cell consisting of a receptor cell and donor cell was used for trans-corneal permeation studies. Out of several developed Formulations, Formulation code A was selected on the basis of in vitro release studies, and was subjected to trans-corneal permeation studies through a goat's corneal membrane. The developed thermoreversible in situ gel was found to be stable, non-irritant, passing the sterility test, having antimicrobial activity and sustained drug release profile.
\end{abstract}

Key words: Azithromycin, Thermoresponsive, Ocular, In situ, corneal permeation

\section{INTRODUCTION}

Several approaches have been tried towards the development of stable sustained release in-situ ophthalmic gel so as to overcome problems associated with conventional ophthalmic dosage form. Conventional ophthalmic drops exhibit a short pre-corneal residence time and poor bioavailability due to rapid and extensive pre-corneal elimination of drugs by solution drainage, lachrymation, and non-productive absorption by conjunctiva. In addition to this, drugs that are hydrophobic or unstable at the $\mathrm{pH}$ of eye fluids cannot be formulated as eye drops. Because of limitations of bioavailability pertaining to ocular route, there are many potent drugs, which still need to be studied for their therapeutic potential by topical ocular route. Novel drug delivery systems such as in situ gels could prove effective approaches of exploring the potential of such drugs. In situ gel system is formulated as liquid preparation suitable to be instilled into eyes which upon exposure to the physiological environment changes to gel results in formation of in situ gel thus increasing the precorneal residence time of the delivery system, and enhances the ocular bioavailability of the drug. ${ }^{1-6}$

Azithromycin, a macrolide antibiotic agent synthetically derived from erythromycin, binds to the $50 \mathrm{~S}$ ribosomal subunit of bacteria and inhibits protein translation and bacterial growth. ${ }^{7,8}$ Laboratory studies of aqueous Azithromycin solutions have shown bactericidal capability against Staphylococcus aureus, Pseudomonas aeruginosa, and respiratory pathogens. ${ }^{9.10}$ In ophthalmology, oral administration of Azithromycin has been proven effective for the treatment of trachoma. The treatment of ocular surface infections with topical Azithromycin is desirable in medicine because systemic exposure to the drug is limited. ${ }^{11}$ Major topical formulation problems arise from the fact that Azithromycin is hydrophobic and sparingly soluble in water at neutral $\mathrm{pH}^{7}$ Therefore, there is a need to design a novel drug delivery system, which could deliver the drug topically. In situ gels are viscous liquids that upon exposure to physiological conditions change into a gel or solid phase in the cul de sac upon its instillation into the eye. In situ gelation approach combines advantages of both solutions and gels, such as accuracy and ease of administration of the solution and prolonged precorneal retention of the gel. ${ }^{12,13}$

Depending on the method employed to cause sol to gel phase transition on the ocular surface, the following three types of systems have been recognized ${ }^{13}$ :

pH-triggered - The polymers used in this system are Pseudolatexes - Carbomer (carbopol), Cellulose acetate phthalate latex (CAP-latex).

Temperature-dependent - Poloxamers (Pluronic, Tetronics), Cellulose derivatives (MC, HPMC), Xyloglucan.

Ion-activated induced - Alginates, Gelrite ${ }^{\circledR}$ (Gellan gum)

For the present work, in situ gels based on thermoreversible Poloxamer 407 polymer were developed.

\section{MATERIALS AND METHODS}

For the present work, in situ gels based on thermoreversible Poloxamer 407 polymer were developed. To develop such a temperature-sensitive gel, the amount required of Poloxamer is very large when it is used alone. Therefore, it is desirable to use other polymer materials along with Poloxamer. By doing this, not only can we reduce the amount of Poloxamer, but also can improve the adhesion of formulation materials to prolong the release of Azithromycin. Hence thermoreversible bioadhesive gels were prepared using poloxamer 407 and different other mucoadhesive polymers such as Sodium Carboxy Methyl cellulose (Na CMC), Hydroxy Propyl Methyl Cellulose (HPMC) K100Lvp, and Polyvinyl Pyrrolidone (PVP) K30. 
Gels were evaluated for physical parameters like appearance, gelation temperature, $\mathrm{pH}$, spreadability, drug content and gel strength ${ }^{14}$.

\section{Preparation of in situ gel}

Thermoreversible mucoadhesive gels of Azithromycin were prepared by cold method as described by Schmolka et $\mathrm{al}^{15}$ using a mechanical stirrer. Accurately weighed quantities of poloxamer and bioadhesive polymers (Table 1), bezalkonium chloride, were dissolved in phosphate buffer of $\mathrm{pH}$ 6.0. To these solutions, the required amount of Azithromycin was added under rapid stirring. The volume was adjusted with phosphate buffer to reach a final concentration of Azithromycin of $1.0 \%(\mathrm{w} / \mathrm{v})$. The solution was mixed well and stored at $4{ }^{\circ} \mathrm{C}$ for overnight which resulted into a clear solution. The developed formulations were filled in 5-ml amber glass vials, closed with gray butyl rubber closures and sealed with aluminum caps. The formulae for the drug formulations are given in Table 1. All gel formulations were evaluated for physical appearance, $\mathrm{pH}$, content uniformity, viscosity, gelation temperature, gel strength, spreadability and invitro drug release.

Based upon the result of in vitro drug release which showed that drug diffusion through the dialysis membrane occurred in the order NaCMC $>$ PVP K30>HPMC K100Lvp, Gel formulation code A with $0.5 \%$ Sodium Carboxy Methyl Cellulose (NaCMC) was selected to carry out stability, microbiological, ocular irritation and permeation studies ${ }^{14}$.

Table 1: Formulation Codes of thermoresponsive bioadhesive gels of Azithromycin

\begin{tabular}{|c|c|c|c|c|c|c|c|c|c|}
\hline \multicolumn{10}{|l|}{ Formula Code } \\
\hline Ingredients & $\mathbf{A}$ & B & $\mathrm{C}$ & D & $\mathbf{E}$ & $\mathbf{F}$ & $\mathbf{G}$ & $\mathbf{H}$ & I \\
\hline Azithromycin(g) & 5.0 & 5.0 & 5.0 & 5.0 & 5.0 & 5.0 & 5.0 & 5.0 & 5.0 \\
\hline Poloxamer 407(g) & 75.0 & 75.0 & 75.0 & 75.0 & 75.0 & 75.0 & 75.0 & 75.0 & 75.0 \\
\hline $\mathrm{NaCMC}(\mathrm{g})$ & 2.5 & 5.0 & 7.5 & - & - & & - & - & - \\
\hline $\begin{array}{l}\text { HPMC } \\
\text { K100Lvp(g) }\end{array}$ & - & - & - & 2.5 & 5.0 & 7.5 & - & - & - \\
\hline PVPK30(g) & - & - & - & - & - & - & 2.5 & 5.0 & 7.5 \\
\hline $\begin{array}{l}\text { Benzalkonium } \\
\text { Chloride } \\
(0.01 \% \mathrm{w} / \mathrm{v}) \\
(\mathrm{g})\end{array}$ & 0.05 & 0.05 & 0.05 & 0.05 & 0.05 & 0.05 & 0.05 & 0.05 & 0.05 \\
\hline $\begin{array}{l}\text { Phosphate Buffer } \\
\text { pH } 6.0 \text { q.s. }\end{array}$ & $500 \mathrm{ml}$ & $500 \mathrm{ml}$ & $500 \mathrm{ml}$ & $500 \mathrm{ml}$ & $500 \mathrm{ml}$ & $500 \mathrm{ml}$ & $500 \mathrm{ml}$ & $500 \mathrm{ml}$ & $500 \mathrm{ml}$ \\
\hline
\end{tabular}

\section{Ocular Permeation Studies across goat's corneal membrane}

Goat's cornea was used for the present investigation to study the permeation across the corneal membrane. Whole eyeballs of goat were procured from a slaughter house and carried to laboratory in cold condition in normal saline maintained at $4 \circ \mathrm{C}$. The cornea were carefully removed and washed with cold normal saline. The washed corneas were kept in cold freshly prepared solution of tear buffer of $\mathrm{pH7}$.4.The study was carried out by using Franz-diffusion cell keeping the corneal side in continuous contact with formulation In the donor compartment. The formulation to be tested was added to the donor chamber with the help of a micropipette. The receptor compartment was filled with Simulated Tear Fluid (STF) of $\mathrm{pH} 7.4$ at $37{ }^{\circ} \mathrm{C} \pm 0.5^{\circ} \mathrm{C}$. The receptor medium was stirred on a magnetic stirrer. The samples were withdrawn at different time intervals and analyzed for drug content. Receptor medium was replaced with an equal volume of STF ( $\mathrm{pH} \mathrm{7.4)} \mathrm{at} \mathrm{each} \mathrm{time} \mathrm{interval} \mathrm{in} \mathrm{order} \mathrm{to} \mathrm{maintain}$ sinking conditions. The samples were appropriately diluted and analyzed. The percent drug released was plotted against time to get the release profile.

\section{Microbiological studies}

The microbiological studies were carried out to ascertain the biological activity of the optimized formulation Code
A and compared with marketed eye drops against microorganism (Staphylococcus aureus) applying 'cup plate method'. A layer of nutrient agar $(20 \mathrm{~mL})$ was seeded with $0.2 \mathrm{~mL}$ of test micro-organism and $7 \mu \mathrm{l}$ of STF and allowed to solidify in the petriplate. Cups were made with the help of sterile borer at $4 \mathrm{~mm}$ diameter on the solidified agar layer. The formulations, $25 \mu \mathrm{l}$ of each were poured into respective cups in an aseptic condition. These plates were kept in incubator for a period of $24 \mathrm{~h}$ and then observed for zone of inhibition. Marketed eye drops of Azithromycin were put in another petri plate for comparison.

\section{Ocular irritation study}

Ocular irritation study of the developed formulation was performed on hen's egg chorioallantoic membrane test which is a rapid, sensitive, and inexpensive test. Testing with an incubated egg is a borderline case between in vivo and in vitro systems and does not conflict with the ethical and legal obligations. The chorioallantoic membrane of the chick embryo is a complete tissue including veins, arteries, and capillaries and is technically very easy to study. It responds to injury with a complete inflammatory process, a process similar to that induced in the conjunctival tissue of the mammals. Developed formulation was tested by this method and the result was compared with those obtained using normal saline, which was used as control that is supposed to be practically non-irritant. A marketed formulation of Azithromycin 1\% Eye drops was also used 
in the ocular irritation studies in comparison with the developed gels. A means score of 0 was obtained for normal saline. The formulation was tested in gel forms A to I. Cumulative score for the gels A to I in gel form were 0 . These scores suggested the formulated gels were practically non-irritant. However, slightly higher but nonsignificant cumulative score (0.9) was obtained in case of marketed eye drops of Azithromycin, which might be due to slightly acidic $\mathrm{pH}$ of the formulation (6.2). Since, a zero cumulative score was obtained for gels, it can be said that the formulated gels were non-irritating.

\section{Sterility testing}

Sterility testing was done as per IP 1996 by employing membrane filtration technique. It was carried out by incubating formulations for not less than 14 days at 30 to $35^{\circ} \mathrm{C}$ in the fluid thioglycolate medium to find the growth of anaerobic bacteria and at 20 to $25^{\circ} \mathrm{C}$ in the soyabean casein digest medium to find the growth of aerobic bacteria and fungi in the formulation.

\section{Stability study}

The formulation code A was selected for the stability study which was conducted according to the International Conference on Harmonization guidelines (ICH). A sufficient quantity of gel solution in glass vials was kept in the oven maintained at temperature of $30 \pm 2{ }^{\circ} \mathrm{C}$ and relative humidity of $60 \pm 5 \%$. The samples were withdrawn at initial, one month, two months and three months' time intervals. The physical parameters of the gel were observed periodically by checking clarity, gel temperature, viscosity and in-vitro drug release profile.

\section{RESULT AND DISCUSSIONS}

\section{Ocular irritation study}

The results of ocular irritation studies showed that the formulated gel was nonirritant without any ocular damage or visible abnormal clinical signs. Therefore the developed in situ gel can be safely instilled into the eye without any adverse effects.

\section{Sterility study}

The optimized formulation passed the test for sterility as there was no appearance of turbidity and hence no evidence of microbial growth when incubated for not less than 14 days a $30-35^{\circ} \mathrm{C}$ in case of fluid thioglycolate medium and at $20-25^{\circ} \mathrm{C}$ in the case of soyabean casein digest medium.

\section{Stability Study}

The stability study was carried out on the formulation code $\mathrm{A}$ at $30 \pm 2{ }^{\circ} \mathrm{C}$ and $60 \pm 5 \% \mathrm{RH}$ for three months. The formulations were clear and no significant changes were observed in gelation temperature, viscosity, in-vitro drug release profile as shown in Table 2.

Table 2: Stability Profile of Formulation Code A

\begin{tabular}{|c|c|c|c|c|}
\hline $\begin{array}{c}\text { Time interval } \\
\text { (Months) }\end{array}$ & $\begin{array}{c}\text { Physical Appearance } \\
\text { of the gel }\end{array}$ & $\begin{array}{c}\text { Gel temperature } \\
\left({ }^{\circ} \mathbf{C}\right)\end{array}$ & $\begin{array}{c}\text { Viscosity (cP) at } \\
\mathbf{2 0} \mathbf{~ r p m ~ a n d ~ 3 7}{ }^{\circ} \mathbf{C}\end{array}$ & $\begin{array}{c}\text { Cumulative \% drug release } \\
\text { At after 5 hours }\end{array}$ \\
\hline Initial & Clear & $\mathbf{3 5 . 6 \pm 0 . 2}$ & $\mathbf{4 9 0 0 \pm 0 . 5}$ & 90.12 \\
\hline 1 & clear & $\mathbf{3 5 . 7 \pm 0 . 2}$ & $4910 \pm \mathbf{0 . 5}$ & 90.13 \\
\hline 2 & clear & $\mathbf{3 5 . 6} \pm \mathbf{0 . 2}$ & $4907 \pm \mathbf{0 . 5}$ & 91.17 \\
\hline 3 & clear & $\mathbf{3 5 . 5} \pm \mathbf{0 . 2}$ & $4920 \pm \mathbf{0 . 5}$ & 90.14 \\
\hline
\end{tabular}

\section{Antimicrobial Activity}

Antimicrobial activity studies showed that diameter of Zone of Inhibition (ZOI) with formulation code A were slightly bigger than that of marketed formulation of Azithromycin which proved that the prepared in situ gel inhibited the growth of Staphylococcus aureus at par with the marketed formulation. The higher ZOI values obtained for the developed gel in comparison to the marketed formulation solution could be attributed to the slow and prolonged diffusion of Azithromycin from polymeric gel due to its high viscosity

Table 3: Antimicrobial activity of the in situ gels against Staphylococcus aureus in cup and plate technique

\begin{tabular}{|c|c|}
\hline In situ gel formulation & Zone of Inhibition(mm) \\
\hline Formulation Code A & $28 \pm 0.30$ \\
\hline $\begin{array}{c}\text { Marketed Formulation of } \\
\text { Azithromycin }\end{array}$ & $32 \pm 0.40$ \\
\hline
\end{tabular}

\section{Ex vivo corneal permeation studies}

Trans-corneal permeation studies were performed using isolated goat's cornea on Franz-diffusion cell using STF
(pH 7.4) at $37 \pm 0.5^{\circ} \mathrm{C}$. The samples were withdrawn at regular intervals and analyzed for drug content. The percent drug released was plotted against time to get drug release profile. Table 4 shows the percent drug release and Fig. 1 shows the percent drug release.

Table 4: Drug Release profile of Formulation code A on isolated goat's cornea

\begin{tabular}{|c|c|}
\hline Time (hrs) & \% Drug Release \\
\hline 0 & 0.00 \\
\hline 1 & 41.00 \\
\hline 2 & 52.03 \\
\hline 3 & 65.03 \\
\hline 4 & 76.30 \\
\hline 5 & 90.09 \\
\hline
\end{tabular}




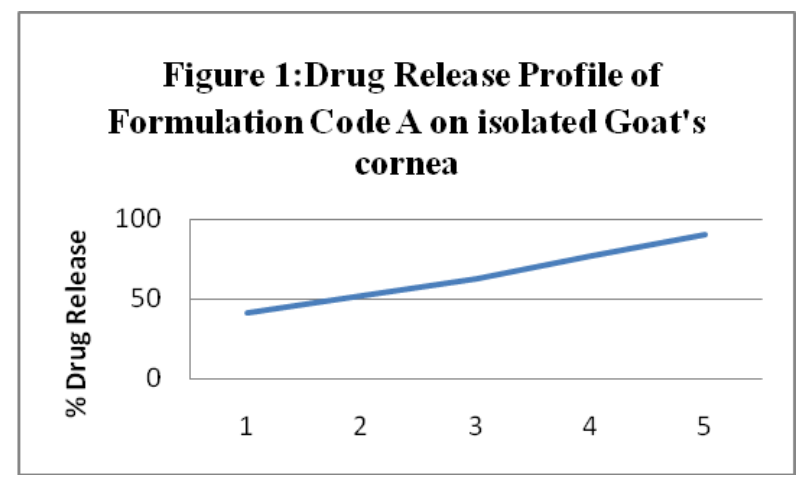

Time (hrs)

\section{CONCLUSION}

The purpose of the present study was to improve the precorneal residence time and sustain the drug release by applying the in situ gelling system approach using Poloxamer in combination with NaCMC, HPMC K100Lvp and PVP K30. It was envisaged that this technique would prove successful for the drug Azithromycin improving its precorneal residence time and sustaining its release. Therefore, the developed in situ gels represent a viable alternative to conventional eye drops. The study showed that a thermoreversible polymer and mucoadhesive polymer can effectively be used to prolong residence time of the drug.

\section{REFERENCES}

1. Kamel A. "In vitro and in vivo evaluation of Pluronic F127-based ocular delivery system for timolol maleate" International Journal of Pharmaceutics. 2002; 241:47-55.

2. Varshosaz J, Tabbakhian M,Salmani Z. "Designing of a Thermosensitive Chitosan/Poloxamer In Situ Gel for Ocular Delivery of Ciprofloxacin" The Open Drug Delivery Journal.2008, 2: 61-70.

3. Kulkarni s , Sandeep N, Shankar S, Ranjit P, Someshwara B,Ramesh B, Ashok Kumar P. "Effect of a single drop of latanoprost ophthalmic gel on intra ocular pressure in the treatment of glaucoma" International Journal of Pharmaceutical Sciences. 2010;2(1):429-435

4. Rathore K. "Insitu Gelling ophthalmic drug delivery: an overview International Journal of Pharmacy and Pharmaceutical Sciences. treatment of glaucoma" International Journal of Pharmaceutical Sciences. 2010;2 Supple 4

5. Ding S. Recent developments in ophthalmic drug delivery. Pharm Sci Technol Today. 1998;1:328-35.

6. Mitra AK, editor. New York: Marcel Dekker; 1993. Ophthal Drug Del Sys; 261-75.

7. Sevillano D, Alou L, Aguilar A, Echevarría O, Giménez MJ, Prieto J. Azithromycin IV pharmacodynamic parameters predicting streptococcus pneumonia killing in epithelial lining fluid versus serum: An in vitro pharmacodynamic situation. J Antimicrob Chemother 2006; 57:1128-33.

8. Retsema J, Fu W. Macrolides: Structures and microbial targets. Int J Antimicrob Agents 2001;18:3-10.

9. Meyer AP, Bril-Bazuin C, Mattie H, Broek VP. Uptake of Azithromycin by human monocytes and enhanced intracellular antibacterial activity against Staphylococcus aureus. Antimicrob Agents Chemother 1993;37:2318-22.

10.Imanura Y, Higashiyama Y, Tomono K, Izumikawa K, Yanagihara K, Ohno H, et al. Azithromycin exhibits bactericidal effects on Psuedomonas aeruginosa through interaction with the outer membrane. Antimicrob Agents Chemother 2005;49:137780.

11. LM, Si E, Pang J, Archibald R, Friedlaender M. Development of a topical polymeric mucoadhesive ocular delivery system for azithromycin. J Ocular Pharmacol Therap. 2009;25:2.

12. Bourlais CL, Needham T, Leverge R. Ophthalmic drug delivery systems. Prog Retin Eye Res 1998;17:33-8.

13. Nanjawade BK, Manvi FV, Manjappa AS. In situ forming hydrogels for sustained ophthalmic drug delivery. J Control Release 2007;122:119-34

14. Bhatia HB, Sachan A, Bhandari A, Formulation and evaluation of ins situ gel for sustained ocular delivery of Azithromycin. Int J Uni Pharm Bio Sci 2013;2(4)639-647.

15. Schmolka IR, A review of block polymer surfactants. J Am Oil Chem Soc. 1977; 54:110-116. 